CASE REPORT

\title{
Hepatitis $C$ virus genotype testing in paraffin wax embedded liver biopsies for specimen identification
}

\author{
Y Ikura, M Ohsawa, E Hai, H Jomura, M Ueda
}

J Clin Pathol 2003;56:960-962

Despite advances in medical technology, careful specimen identification is still a fundamental principle of laboratory testing. If pathological samples are mixed up, especially in the case of extremely small biopsy samples, large amounts of time and energy may be wasted in correctly identifying the specimens. Recently, two liver biopsy specimens were mixed up in this department, and a new pathological technology was used to resolve the issue. Liver biopsy was performed on two patients with hepatitis $\mathrm{C}$ virus $(\mathrm{HCV})$ infection. During sample transfer or tissue processing, the biopsy specimens were mixed up. Because the $A B O$ blood group of the two patients was identical (type $A B$ ), the specimens were subsequently identified by analysing the HCV genotypes. RNA extracted from the paraffin wax embedded liver specimens was examined by a polymerase chain reaction based HCV genotype assay. This enabled the correct identification of the specimens, and each patient received the appropriate treatment on the basis of the accurate diagnosis.

A a result of advances in molecular biotechnologies, various gene assays can now be performed on paraffin wax embedded pathological specimens. ${ }^{1}$ Both RNA and DNA can be extracted from paraffin wax blocks and then analysed by the polymerase chain reaction (PCR). ${ }^{2}$ However, this technique is used mainly as a research tool, and its clinical uses are limited.

Careful specimen identification is unequivocally a fundamental principle of pathological examination. ${ }^{3}$ If extremely small biopsy specimens are mixed up, large amounts of time and effort can be wasted in correctly identifying them. Previous reports have demonstrated the value of new technologies in correctly identifying pathological specimens that have been mixed up. ${ }^{4-7}$ Gene assays can be extremely useful for sorting out these situations. ${ }^{5}$

Here, we report our experience of a mix up with two liver biopsy specimens. Genotyping of hepatitis $\mathrm{C}$ virus (HCV) RNA was performed on the paraffin wax embedded specimens, and easily resolved the situation.

\section{CASE REPORT}

Two female patients with HCV infection received ultrasonography guided liver biopsy on the same day. Their clinical courses are as follows.

\section{Patient A}

Patient A was a 31 year old haemodialysed woman with chronic renal failure. Five years before the liver biopsy was taken, she was diagnosed as having HCV (genotype Ib) infection, although her liver function was normal. We performed a liver biopsy to make a detailed analysis of her liver. The biopsy specimen ( sample A) was transferred to our

\section{Take home messages}

- Viral genome analysis is a useful method for identifying mislabelled pathological specimens

- Such analysis is particularly useful when patients have the same characteristics, such as sex and $A B O$ blood group

pathology laboratory, and a diagnosis of chronic active hepatitis with severe fibrosis (fig lA) was made. Consequently, her physician wished to start treatment with interferon.

\section{Patient B}

Patient B was a 61 year old woman with advanced chronic HCV (genotype IIa) hepatitis, who had been treated once with interferon but failed to eradicate the virus, and had been recommended by her physician to receive an additional treatment with interferon. We performed the liver biopsy to check her liver condition (sample B). The pathology report commented that she had chronic hepatitis, but only mild necroinflammatory activity and fibrosis (fig 1B), so that interferon treatment was postponed.

\section{Resolving the problem}

Because there seemed to be a discrepancy between the clinical and pathological findings of these two patients, we considered the possibility of a mix up of the specimens. To check the identity of the specimens, we intended to identify their ABO blood group antigens using immunohistochemistry, but their blood group was identical (type $A B$ ). The physicians had noted that they were both infected by different genotypes of HCV, so we decided to use HCV genotyping to identify the samples.

\section{METHODS}

Total RNA was extracted from the paraffin wax embedded liver specimens as described previously. ${ }^{8}$ The RNA samples were tested by nested PCR based HCV genotyping. After the first amplification using primers compatible with all HCV genotypes, the second PCR was done with a mixture of genotype specific primer sets (genotype Ib, 5'-AGGAAGACTTCCGAGCGGTC and 5'GAGCCATCCTGCCCACCCCA; genotype IIa, 5'-AGGAAGAC TTCCGAGCGGTC and 5'-CCAAGAGGGACGGGAACCCT). The sizes of the amplicons were $144 \mathrm{bp}$ for genotype Ib and $174 \mathrm{bp}$ for genotype IIa. Because the genotypes of HCV in each patient had been determined previously, the sera sampled on the day before the biopsy were simultaneously tested as positive controls.

Abbreviations: $\mathrm{HCV}$, hepatitis $\mathrm{C}$ virus; $\mathrm{PCR}$, polymerase chain reaction 

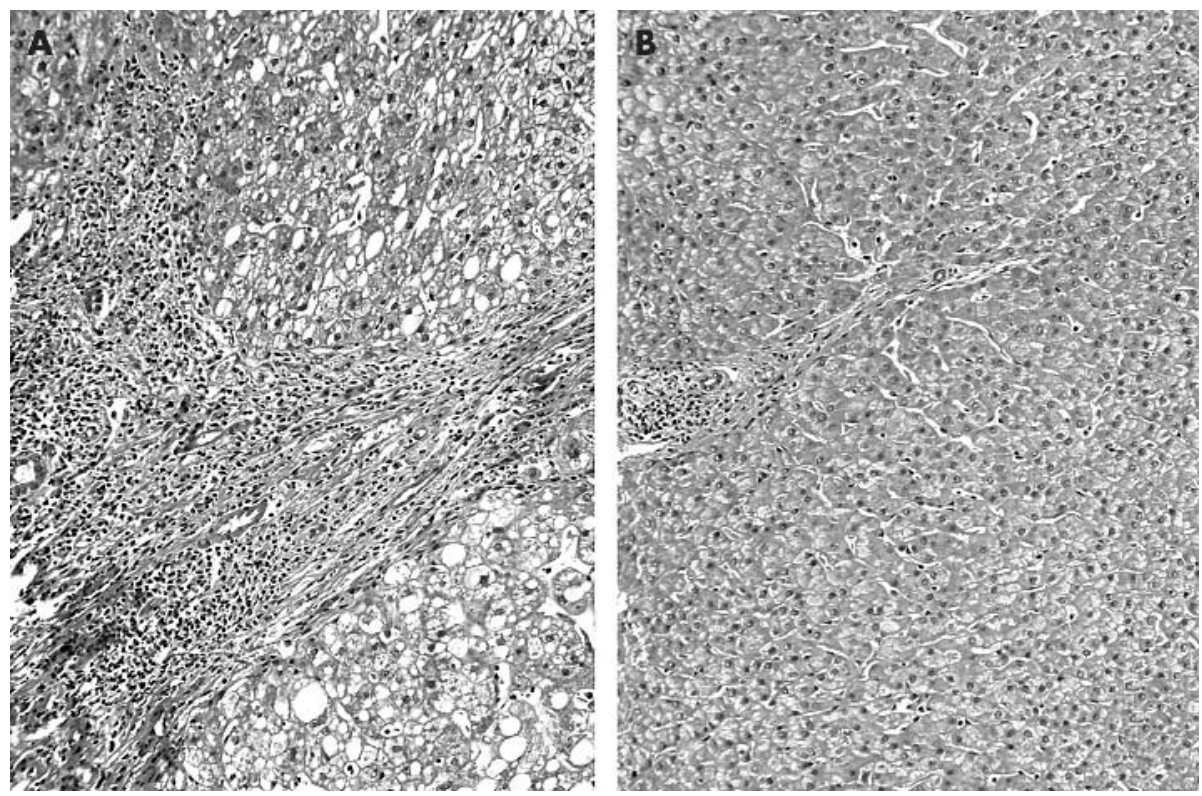

Figure 1 Histological findings of the liver biopsy specimens. (A) Sample A: the portal area shows pronounced lymphoid infiltration with piecemeal necrosis and bridging fibrosis. (B) Sample B: the liver tissue shows mild portal inflammation without evident necroinflammatory reaction or fibrosis.

\section{RESULTS}

The HCV genotype assay detected genotype IIa in sample A and the serum from patient $\mathrm{B}$; in contrast, genotype $\mathrm{Ib}$ was detected in sample $\mathrm{B}$ and the serum from patient $\mathrm{A}$ (fig 2). Samples A and B were negative for genotypes Ib and IIa, respectively. It is known that the HCV genotype is identical in liver tissue and serum, except in multigenotype infections. ${ }^{9}$ Hence, we concluded that the liver samples had been mislabelled: sample A was from patient $\mathrm{B}$ and sample B was from patient A. The pathological diagnoses were corrected as follows: patient A had chronic hepatitis $\mathrm{C}$ with minimal activity and patient $\mathrm{B}$ had chronic active hepatitis with severe fibrosis. Although the reason for the mix up was not clear, we informed the patients of the results, both of whom accepted the correction and our explanation. Interferon treatment was soon initiated in patient B.

\section{DISCUSSION}

Various techniques have been used for specimen identification. ${ }^{4-7}$ Immunohistochemistry for ABO blood group antigens is the most frequently used method, ${ }^{4}$ but sex chromosomes and HLA antigens are also useful markers. ${ }^{5}{ }^{6}$ However, ABO antigen testing and sex chromosome identification cannot

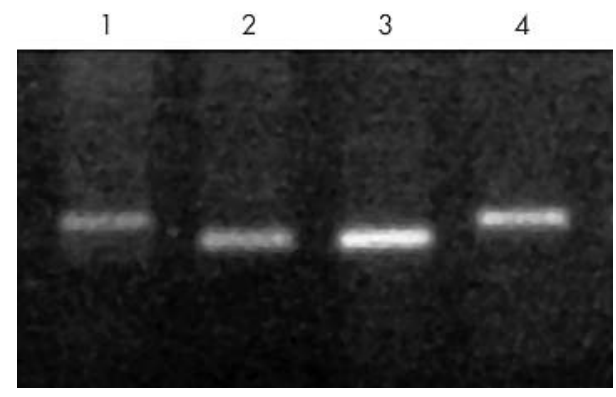

Figure 2 Results of hepatitis $C$ virus genotyping. The polymerase chain reaction assay detected genotype lla in sample $A$ and serum from patient $B$, and genotype $\mathrm{lb}$ in sample $B$ and serum from patient $A$. Lane 1 , serum from patient $B$ (positive control for genotype lla, 174 bp); lane 2, serum from patient $A$ (positive control for genotype $\mathrm{lb}, 144 \mathrm{bp}$ ); lane 3, sample B (genotype Ib); lane 4, sample A (genotype lla). be used if patients have the same characteristics. For HLA antigen testing, additional blood tests are needed to clarify the patient's HLA type. Thus, other methods have been sought to resolve these rare but serious problems.

"Similar accidents to the one reported here are likely to occur in the future, and our method may be useful for uncovering the correct specimen identification"

DNA genotyping, a recently developed molecular biological technique, can also be used for specimen identification. $^{7}$ However, it is a complex test that requires specialised reagents. Therefore, we used our standard laboratory test, HCV genotyping, which we can do easily and accurately. Soguero et al have used this technique as a research tool to carry out HCV genotyping in paraffin wax embedded pathology specimens. ${ }^{10}$ Our present case report demonstrates the usefulness of this technique in a clinical setting. In Japan, there are many patients with chronic viral hepatitis, and liver biopsy will probably be performed more frequently in the future. Similar accidents to the one reported here are likely to occur in the future, and our method may be useful for uncovering the correct specimen identification. Although they are not the patient's own genes, viral genomes can be very useful as identification markers.

Our experience may be an exceptional one and our method may not be suited to all pathological laboratories. However, we would like to emphasise that advances in medical technology should be used in the clinical setting and should provide direct benefits to patients.

\section{Authors' affiliations}

Y Ikura, M Ohsawa, E Hai, M Ueda, Department of Pathology, Osaka City University Graduate School of Medicine, 1-4-3, Asahimachi, Abeno-ku, Osaka 545-8585, Japan

H Jomura, Department of Internal Medicine, Wakakokai Hospital, Osaka, Japan

Correspondence to: Dr Y Ikura, Department of Pathology, Osaka City University Graduate School of Medicine, 1-4-3, Asahimachi, Abeno-ku, Osaka 545-8585, Japan; ikura@med.osaka-cu.ac.jp

Accepted for publication 3 June 2003 


\section{REFERENCES}

1 Crisan D, Mattson JC. Retrospective DNA analysis using fixed tissue specimens. DNA Cell Biol 1993;12:455-64.

2 Dakhama A, Macek V, Hogg JC, et al. Amplification of human beta-actin gene by the reverse transcriptase-polymerase chain reaction: implications for assessment of RNA from formalin-fixed, paraffin-embedded material. J Histochem Cytochem 1996;44:1205-7.

3 Nakhleh RE, Zarbo RJ. Surgical pathology specimen identification and accessioning: a College of American Pathologists Q-probes study of 1,004,115 cases from 417 institutions. Arch Pathol Lab Med 1996; 120:227-33.

4 Ritter JH, Sutton TD, Wick MR. Use of immunostains to ABH blood group antigens to resolve problems in identity of tissue specimens. Arch Pathol Lab Med 1994; 1 18:293-7.

5 Kibbelaar RE, Leenheers-Binnendijk CF, Spaander PJ, et al. Biopsy specimen identification by detection of sex chromosomes: application of in situ hybridisation. J Clin Pathol 1992;45:149-50.
6 Lagaaij EL, Cramer-Knijnenburg GF, Van der Pij JW, et al. Rapid verification of the identity of questionable specimens using immunohistochemistry with monoclonal antibodies directed against HLA-class I antigens. Histopathology 1997:31:284-8.

7 Shibata D, Namiki T, Higuchi R. Identification of a mislabeled fixed specimen by DNA analysis. Am J Surg Pathol 1990;14:1076-8.

8 Ogami M, Ikura Y, Nishiguchi S, et al. Quantitative analysis and in situ localization of human telomerase RNA in chronic liver disease and hepatocellular carcinoma. Lab Invest 1999;79:15-26.

9 Fan X, Solomon H, Poulos JE, et al. Comparison of genetic heterogeneity of hepatitis C viral RNA in liver tissue and serum. Am J Gastroenterol 1999:94:1347-54.

10 Soguero C, Campo E, Ribalta T, et al. Assessment of genotype and molecular evolution of hepatitis $C$ virus in formalin-fixed paraffin-embedded liver tissue from patients with chronic hepatitis $C$ virus infection. Lab Invest 2000;80:851-6. 